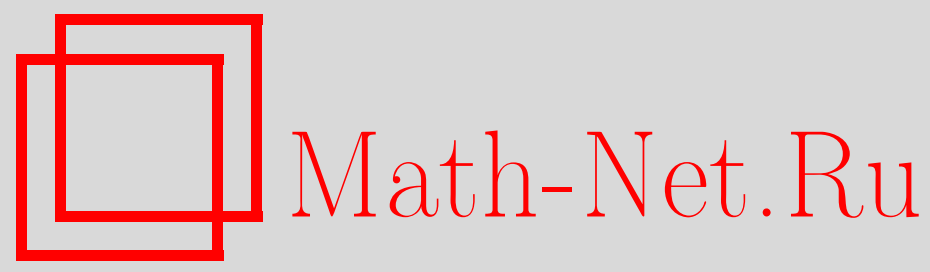

В. И. Масол, Некоторые применения оценки мощности одной конфигурации, Матем. заметки, 1998, том 63, выпуск 5, 729-736

DOI: https://doi.org/10.4213/mzm1340

Использование Общероссийского математического портала Math-Net.Ru подразумевает, что вы прочитали и согласны с пользовательским соглашением http://www.mathnet.ru/rus/agreement

Параметры загрузки:

IP: 54.92 .164 .108

26 апреля 2023 г., $14: 31: 36$

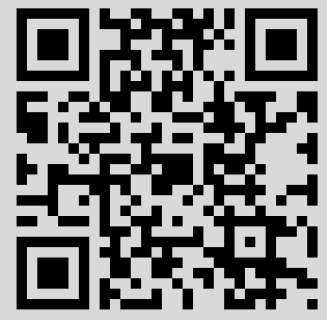




\section{НЕКОТОРЫЕ ПРИМЕНЕНИЯ ОЦЕНКИ МОЩНОСТИ ОДНОЙ КОНФИГУРАЦИИ}

\section{В.И. Масол}

Строятся две конфигурации и мощность одной из них оценивается через мощность другой. Полученная оценка применяется к некоторым комбинаторным задачам.

Библиография: 3 названия.

0. Пусть $W$ - совокупность всех непустых подмножеств конечного множества $\Omega$, мощность которого $|\Omega|=k, 1 \leqslant k<\infty$. Рассмотрим две конфигурации (определение понятия “конфигурация" см. в $[1$, с. 34$]) W_{\Delta}$ и $I_{s}$, где

$$
\begin{gathered}
W_{\Delta} \subseteq W, \quad W_{\Delta}=\left\{\omega_{1}, \ldots, \omega_{\Delta}\right\}, \quad\left|W_{\Delta}\right|=\Delta, \quad \Delta \geqslant 1, \\
\omega_{i} \neq \omega_{j} \quad \text { для } i \neq j, \quad i, j \in\{1, \ldots, \Delta\} \\
I_{s} \subseteq W, \quad I_{s}=\left\{m_{1}, \ldots, m_{s}\right\}, \quad\left|I_{s}\right|=s, \quad s \geqslant 0, \\
m_{i} \neq m_{j} \quad \text { для } i \neq j, \quad i, j \in\{1, \ldots, s\}
\end{gathered}
$$

удовлетворяющие условию

$$
\left|m_{i} \cap \omega_{j}\right| \equiv 0(\bmod 2), \quad i=\overline{1, s}, \quad j=\overline{1, \Delta} .
$$

В п. 1 дана оценка мощности множества $I_{s}$ при выполнении условий (1) и

$$
\Delta \in\left[2^{r-1}, 2^{r}-1\right], \quad 1 \leqslant r \leqslant k .
$$

Полученная оценка используется при решении некоторых комбинаторных задач типа перечислительных (пп. 2,3), существования и построения (п. 4) (определения указанных типов задач см., например, в $[1$, с. 36$])$.

1. Введем некоторые обозначения. Пусть $L_{\alpha_{1}, \ldots, \alpha_{z}}$ - совокупность всех элементов, которые принадлежат $\omega_{\alpha_{1}}, \ldots, \omega_{\alpha_{z}}$ и только, $\left|L_{\alpha_{1}, \ldots, \alpha_{z}}\right|=l_{\alpha_{1}, \ldots, \alpha_{z}}$, где $1 \leqslant \alpha_{1}<\cdots<$ $\alpha_{z} \leqslant \Delta, z=\overline{1, \Delta}$. Обозначим через $\varphi$ общее количество положительных (непустых) элементов множества $\left\{l_{\alpha_{1}, \ldots, \alpha_{z}}: 1 \leqslant \alpha_{1}<\cdots<\alpha_{z} \leqslant \Delta, z=\overline{1, \Delta}\right\}\left(\left\{L_{\alpha_{1}, \ldots, \alpha_{z}}: 1 \leqslant\right.\right.$ $\left.\left.\alpha_{1}<\cdots<\alpha_{z} \leqslant \Delta, z=\overline{1, \Delta}\right\}\right)$. Число $\varphi$ назовем характеристическим числом конфигураиии $W_{\Delta}$. Далее, компоненты вектора $\vec{x}(v)=\left(x_{1}(v), \ldots, x_{\varphi}(v)\right), v \in W$, будем строить по следующему правилу:

$$
x_{t}(v)=\left\{\begin{array}{l}
1, \text { если }\left|v \cap L_{k_{t}}\right| \equiv 1(\bmod 2), \\
0, \text { если }\left|v \cap L_{k_{t}}\right| \equiv 0(\bmod 2),
\end{array}\right.
$$


где $t=\overline{1, \varphi}, k_{1}, \ldots, k_{\varphi}$ - попарно различные элементы множества

$$
\left\{\left\{\alpha_{1}, \ldots, \alpha_{z}\right\}: 1 \leqslant \alpha_{1}<\cdots<\alpha_{z} \leqslant \Delta, z=\overline{1, \Delta}\right\},
$$

каждому из которых соответствует положительное (непустое) $l_{k_{1}}, \ldots, l_{k_{\varphi}}\left(L_{k_{1}}, \ldots\right.$, $\left.L_{k_{\varphi}}\right)$. Введем, наконец, однородную систему линейных уравнений

$$
\sum_{t=1}^{\varphi} a_{q}^{(t)} x_{t}=0, \quad q=\overline{1, \Delta}
$$

над полем $\mathrm{GF}(2)$ с матрицей коэффициентов $A$, содержащей $\Delta$ строк и $\varphi$ столбцов,

$$
A=\left\|a_{q}^{(t)}\right\|_{q=\overline{1, \Delta}}^{t=\overline{1, \varphi}}, \quad \text { где } \quad a_{q}^{(t)}=\left\{\begin{array}{ll}
1, & \text { если } q \in k_{t}, \\
0, & \text { если } q \notin k_{t},
\end{array} \quad t=\overline{1, \varphi}, \quad q=\overline{1, \Delta .}\right.
$$

Матрицу $A$ назовем матрицей, построенной на конфигурации $W_{\Delta}$.

ТЕОРема 1. Пусть выполняются условия (1) $и$ (2). Тогда

$$
s \leqslant 2^{k-r}-1 \text {. }
$$

ДокАЗАТЕЛЬСтво. Если $s=0$, то, очевидно, (5) выполняется. Пусть $s>0$. С помощью правила (3) для произвольного $\widetilde{m} \in I_{s}$ можно построить вектор $\vec{x}(\widetilde{m})$, которьй является решением системы (4). В самом деле, из $\widetilde{m} \in I_{s}$ в силу (1) следует, что $\left|\widetilde{m} \cap \omega_{j}\right| \equiv 0(\bmod 2), j=\overline{1, \Delta}$, но

$$
\omega_{j}=\bigcup_{t=1}^{\varphi} L_{k_{t}}^{(j)}, \quad \text { где } L_{k_{t}}^{(j)}= \begin{cases}L_{k_{t}}, & \text { если } a_{j}^{(t)}=1, \\ \varnothing, & \text { если } a_{j}^{(t)}=0 .\end{cases}
$$

Значит,

$$
\left|\widetilde{m} \cap \omega_{j}\right|=\sum_{t=1}^{\varphi}\left|\widetilde{m} \cap L_{k_{t}}^{(j)}\right|, \quad j=\overline{1, \Delta},
$$

откуда $a_{j}^{(1)} x_{1}(\widetilde{m}) \oplus \cdots \oplus a_{j}^{(\varphi)} x_{\varphi}(\widetilde{m})=0, j=\overline{1, \Delta}$, где символ “ $\oplus$ ” обозначает сложение по модулю 2.

Будем обозначать совокупность всех элементов множества $I_{s}$, соответствующих построенному с помошю правила (3) решению $\vec{x}(\widetilde{m})$ системы (4), где $\widetilde{m} \in I_{s}$, через $M(\widetilde{m})$, т.е. $M(\widetilde{m}) \subseteq I_{s}, M(\widetilde{m})=\left\{v_{1}, \ldots, v_{\mu}\right\}, v_{i} \neq v_{j}$ для $i \neq j, i, j \in\{1, \ldots, \mu\} ; \vec{x}\left(v_{t}\right)=\vec{x}(\widetilde{m})$ для $t=\overline{1, \mu} ; \vec{x}(v) \neq \vec{x}(\widetilde{m})$ для $v \in I_{s} \backslash M(\widetilde{m})$. Пусть вектор $\vec{x}(\widetilde{m})$ содержит $\varkappa, 0 \leqslant \varkappa \leqslant \varphi$, единичных компонент, расположенных на местах с номерами $i_{1}, \ldots, i_{\varkappa}$ и $\varphi-\varkappa$ нулевых компонент, расположенных на местах с номерами $j_{1}, \ldots, j_{\varphi-\varkappa}$. Тогда, очевидно, мощность $\mu$ множества $M(\widetilde{m})$ удовлетворяет неравенству

$$
\mu \leqslant 2^{k-s_{1}+s_{2}+s_{3}}-\chi(\varkappa=0)
$$

где $s_{1}=\sum_{t=1}^{\varphi} l_{k_{t}}, 2^{k-s_{1}}-$ количество всех подмножеств множества $\Omega \backslash \bigcup_{t=1}^{\varphi} L_{k_{t}}$;

$$
s_{2}=\sum_{i \in\left\{i_{1}, \ldots, i_{\varkappa}\right\}}\left(l_{k_{i}}-1\right)
$$


$2^{l_{k_{i}}-1}$ - количество всех подмножеств множества $L_{k_{i}}, i \in\left\{i_{1}, \ldots, i_{\varkappa}\right\}$, каждое из которых содержит нечетное число элементов из $\Omega$;

$$
s_{3}=\sum_{j \in\left\{j_{1}, \ldots, j_{\varphi-\varkappa}\right\}}\left(l_{k_{j}}-1\right),
$$

$2^{l_{k_{j}}-1}$ - количество всех подмножеств множества $L_{k_{j}}, j \in\left\{j_{1}, \ldots, j_{\varphi-\varkappa}\right\}$, каждое из которых содержит четное число элементов из $\Omega$;

$$
\chi(\varkappa=0)= \begin{cases}1, & \text { если } \varkappa=0, \\ 0, & \text { если } \varkappa \neq 0 .\end{cases}
$$

Из (6) и определений сумм $s_{1}, s_{2}, s_{3}$ следует $\mu \leqslant 2^{k-\varphi}-\chi(\varkappa=0)$. Итак, каждому $\widetilde{m} \in I_{s}$ соответствует построенное с помошью правила (3) решение системы (4) и число элементов из $I_{s}$, соответствующих этому решению, не превосходит $2^{k-\varphi}-\chi(\varkappa=0)$. Отсюда, очевидно, получаем

$$
s \leqslant 2^{k-\varphi} R-1,
$$

где $R$ - число решений системы (4). В силу (2) ранг $f$ матрицы $A$ коэффициентов системы $(4), \operatorname{rank}(A)=f$, над полем $\mathrm{GF}(2)$ удовлетворяет неравенству $f \geqslant r$. Поэтому $R=2^{\varphi-f} \leqslant 2^{\varphi-r}$. Отсюда с учетом (7) имеем (5). Теорема 1 доказана.

ЗАмЕчАнИЕ 1. Из доказательства теоремы 1 следует, что если имеют место (1), (2) и $f>r$, то $s<2^{k-r}-1$. Поэтому при выполнении условий $(1),(2)$ и $s=2^{k-r}-1$, $1 \leqslant r \leqslant k$, справедливо равенство $\operatorname{rank}(A)=r$.

2. Обозначим через $H(\omega), \omega \in W$, конфигурацию, содержащую все такие попарно различные элементы множества $W$, что для произвольного $v \in H(\omega)$ выполняется соотношение $|v \cap \omega| \equiv 1(\bmod 2)$. Пусть $v \in H\left(\omega_{i_{1}}\right)$, где $i_{1} \in\{1, \ldots, \Delta\}$, и $T$ - число всех конфигураций $H\left(\omega_{i_{1}}\right), \ldots, H\left(\omega_{i_{T}}\right)$ среди $H\left(\omega_{j}\right), j \in \overline{1, \Delta}$, каждая из которых содержит элемент $v$ такой, что $v \in H\left(\omega_{i_{\nu}}\right), i_{\nu} \in\{1, \ldots, \Delta\}, \nu=\overline{1, T}$.

ТЕОРема 2. Если выполняются условия (1) $и$

$$
\Delta=2^{r}-1, \quad s=2^{k-r}-1, \quad 1 \leqslant r \leqslant k,
$$

mo $T=2^{r-1}$.

ДоказАтЕльство. Пусть матрица $A$ построена на конфигурации $W_{\Delta}$, вектор $\vec{x}(v)$ построен с помощью правила (3). Отсюда в силу определения конфигурации $H\left(\omega_{i_{1}}\right)$ имеем для $v \in H\left(\omega_{i_{1}}\right)$

$$
a_{i_{1}}^{(1)} x_{1}(v) \oplus \cdots \oplus a_{i_{1}}^{(\varphi)} x_{\varphi}(v)=1
$$

Условия $(1),(8)$ и замечание 1 позволяют заключить, что $\operatorname{rank}(A)=r$. Путем подходящих замен можно добиться того, чтобы $i_{1}$-я строка матрицы $A$ находилась среди $r$ линейно независимых над $\mathrm{GF}(2)$ строк этой же матрицы $A$. Пусть указанные $r$ строк имеют номера $z_{1}, \ldots, z_{r}$, причем первые $\gamma, 1 \leqslant \gamma \leqslant r$, среди них таковы, что

$$
a_{\mu}^{(1)} x_{1}(v) \oplus \cdots \oplus a_{\mu}^{(\varphi)} x_{\varphi}(v)=d
$$


где $d \in \mathrm{GF}(2)$ и

$$
d=\left\{\begin{array}{l}
1, \text { если } \mu \in\left\{z_{1}, \ldots, z_{\gamma}\right\}, \\
0, \text { если } \mu \in\left\{z_{1}, \ldots, z_{r}\right\} \backslash\left\{z_{1}, \ldots, z_{\gamma}\right\} .
\end{array}\right.
$$

(В силу (9) и выбора номеров $z_{1}, \ldots, z_{r}$ имеем $i_{1} \in\left\{z_{1}, \ldots, z_{\gamma}\right\}$, поэтому $\gamma \geqslant 1$.) Тогда все ненулевые линейные комбинации $r$ линейно независимых над $\mathrm{GF}(2)$ строк матрищы $A$ дадут $\Delta$ равенств

$$
a_{j}^{(1)} x_{1}(v) \oplus \cdots \oplus a_{j}^{(\varphi)} x_{\varphi}(v)=d, \quad j=\overline{1, \Delta}
$$

в правых частях которых единица встретится $2^{r-1}$ раз. Действительно, $2^{\gamma-1}$ единиц получается в результате всевозможных линейных комбинаций первых $\gamma$ строк матрицы $A$. Линейные комбинации строк матрицы $A$ с номерами $\gamma+1, \gamma+2, \ldots, r$ дадут $2^{r-\gamma}-1$ строк матрищы $A$, которые в сумме с каждой из $2^{\gamma-1}$ уже построенных строк приводят к равенству в системе (10) с единицей в правой части. Значит, в системе (10) обшее число равенств с единищей в правой части равно $\left(2^{r-\gamma}-1\right) 2^{\gamma-1}+2^{\gamma-1}=2^{r-1}$.

Если для произвольного $j \in\{1, \ldots, \Delta\}$ в правой части (10) $d=1$, то в силу построений матрицы $A$ и вектора $\vec{x}(v)$ имеем $\left|v \cap \omega_{j}\right| \equiv 1(\bmod 2)$, отсюда $v \in H\left(\omega_{j}\right)$; а если $d=0$, то $\left|v \cap \omega_{j}\right| \equiv 0(\bmod 2)$, т.е. $v \notin H\left(\omega_{j}\right)$. Теорема 2 доказана.

3. Рассмотрим $\Delta+1$ не совпадаюших между собой конфигураций $H\left(\omega_{j}\right), j=\overline{1, \Delta+1}$, и обозначим через $H$ совокупность всех элементов $h \in H\left(\omega_{\Delta+1}\right)$, для которых $h \notin H\left(\omega_{j}\right)$, $j=\overline{1, \Delta}$. Прежде чем сформулировать теорему 3 о мощности множества $H$, докажем лемму 1. Пусть система уравнений

$$
\sum_{t=1}^{\psi} a_{q}^{(t)} x_{t}=0, \quad q=\overline{1, \Delta}, \quad \sum_{t=1}^{\psi} a_{\Delta+1}^{(t)} x_{t}=1
$$

над полем $\mathrm{GF}(2)$ имеет матрицу коэффициентов $\widetilde{A}=\left\|a_{q}^{(t)}\right\|_{q=\overline{1, \Delta+1}}^{t=\overline{1, \psi}}$, построенную на $W_{\Delta+1}$; здесь $\psi$ - характеристическое число конфигурации $W_{\Delta+1}$.

ЛЕмМа 1. Если выполняются условия (1) $и$

$$
\Delta=2^{r}-1, \quad s=2^{k-r}-1, \quad 1 \leqslant r \leqslant k-1,
$$

то число $R$ решений системы (11) удовлетворяет равенству

$$
R=2^{\psi-r-1}
$$

ДокАЗАТЕЛЬСТво. Покажем, что

$$
\operatorname{rank}(\widetilde{A})=r+1
$$

Пусть $c_{t}-$ совокупность всех элементов множества $\omega_{\Delta+1}, c_{t} \subseteq \omega_{\Delta+1}$, таких, что $c_{t} \subseteq L_{k_{t}}, t=\overline{1, \varphi}$, где $\varphi-$ характеристическое число конфигурации $W_{\Delta}$, и $c_{0} \subseteq \omega_{\Delta+1}$, $c_{0} \cap \omega_{j}=\varnothing, j=\overline{1, \Delta}$. Исходя из матрицы $A$, введенной в п. 1 , можно построить с 
точностью доперестановки строк и столбцов матрицу $\widetilde{A}$, воспользовавшись следующим алгоритмом. Если для некоторого $t \in\{1, \ldots, \varphi\} c_{t}=\varnothing\left(c_{t}=L_{k_{t}}\right)$, то столбец

$$
\left(\begin{array}{c}
a_{1}^{(t)} \\
\vdots \\
a_{\Delta}^{(t)}
\end{array}\right)
$$

матрицы $A$, дополненньй элементом $a_{\Delta+1}^{(t)}=0\left(a_{\Delta+1}^{(t)}=1\right)$, является столбцом матрицы $\widetilde{A}$. Если для указанного $t c_{t} \subset L_{k_{t}}, c_{t} \neq \varnothing$, то столбцы

$$
\left(\begin{array}{c}
a_{1}^{(t)} \\
\vdots \\
a_{\Delta}^{(t)} \\
1
\end{array}\right) \quad \text { и } \quad\left(\begin{array}{c}
a_{1}^{(t)} \\
\vdots \\
a_{\Delta}^{(t)} \\
0
\end{array}\right)
$$

являются столбцами матрицы $\widetilde{A}$. Наконец, если $c_{0} \neq \varnothing$, то столбец

$$
\left(\begin{array}{c}
0 \\
\vdots \\
0 \\
1
\end{array}\right) \Delta \text { paз }
$$

также является столбцом матрицы $\widetilde{A}$. Нетрудно понять, что иных столбцов матрища $\widetilde{A}$ иметь не может.

Отсюда следует, что если $\psi>\varphi$ и $c_{0} \neq \varnothing$, то матрицу $\widetilde{A}$ можно представить в виде

$$
\widetilde{A}=\left(\begin{array}{cccc}
0 & & & \\
\vdots & & A^{*} & \\
0 & & & \\
1 & a_{\Delta+1}^{(1)} & \ldots & a_{\Delta+1}^{(\psi-1)}
\end{array}\right)
$$

где $A^{*}$ - матрица, имеющая $\Delta$ строк и $\psi-1$ столбцов, которая либо совпадает с $A$, либо отличается от $A$ тем, что содержит совпадающие между собой столбцы. В силу $(1),(12)$ и замечания $1 \operatorname{rank}(A)=r$. Значит, $\operatorname{rank}\left(A^{*}\right)=r$. Отсюда $\operatorname{rank}(\widetilde{A})=r+1$, т.е. (14) выполняется.

Если $\psi>\varphi$ и $c_{0}=\varnothing$, то аналогично (15) получаем

$$
\widetilde{A}=\left(\begin{array}{ccc} 
& & \\
& \widetilde{A}^{*} & \\
& & \\
a_{\Delta+1}^{(1)} & \ldots & a_{\Delta+1}^{(\psi)}
\end{array}\right),
$$

где $\widetilde{A}^{*}$ - матрица размера $\Delta \times \psi$, отличающаяся от $A$ лишь тем, что содержит совпадающие между собой столбцы. Пусть среди них будут, в частности, столбцы с номерами $\xi$ и $\zeta$, где $\xi, \zeta \in\{1, \ldots, \psi\}$. В таком случае, либо $a_{\Delta+1}^{(\xi)}=1$ и $a_{\Delta+1}^{(\zeta)}=0$, либо $a_{\Delta+1}^{(\xi)}=0$ 
и $a_{\Delta+1}^{(\zeta)}=1$. Отсюда, очевидно, имеем $\operatorname{rank}(\widetilde{A})=\operatorname{rank}\left(\widetilde{A}^{*}\right)+1=\operatorname{rank}(A)+1=r+1$, т.е. (14) вьполняется.

Допустим, что $\psi=\varphi$. Тогда

$$
\widetilde{A}=\left(\begin{array}{ccc} 
& & \\
& A & \\
& & \\
a_{\Delta+1}^{(1)} & \ldots & a_{\Delta+1}^{(\psi)}
\end{array}\right)
$$

В силу того, что $\omega_{\Delta+1} \neq \varnothing$, набор $\left(a_{\Delta+1}^{(1)}, \ldots, a_{\Delta+1}^{(\psi)}\right)$ содержит хотя бы одну единицу. Поскольку $\left(a_{\Delta+1}^{(1)}, \ldots, a_{\Delta+1}^{(\psi)}\right) \neq(0, \ldots, 0), \operatorname{rank}(A)=r$ и $\Delta=2^{r}-1$, строка $\left(a_{\Delta+1}^{(1)} \ldots a_{\Delta+1}^{(\psi)}\right)$ в (17) совпадает с одной из строк матрицы $A$. Тогда $\omega_{\Delta+1}$ совпадает с некоторым множеством $\omega_{j}, j \in\{1, \ldots, \Delta\}$, что противоречит выбору $\omega_{1}, \ldots, \omega_{\Delta}, \omega_{\Delta+1}$. Значит, равенство $\psi=\varphi$ не может вьполняться. Соотношение (14) установлено.

На основании (14) и известных результатов (см., например, [2, с. 117-119]) для доказательства (13) достаточно установить совместность системы (11). Если $c_{0} \neq \varnothing$, то с учетом (15) находим, что вектор $(1,0, \ldots, 0)$ является решением системы (11). Если $c_{0}=\varnothing$, то с учетом (16) находим, что $\psi$-мерный вектор, состоящий из $\psi-2$ нулей и двух единиц, расположенных на позициях $\xi$ и $\zeta$, является решением системы (11). Значит, система (11) совместна. Лемма 1 доказана.

ТЕОРема 3. Если выполняются условия (1) $u$ (12), то $|H|=2^{k-r-1}$.

ДоКАЗАТЕЛЬСТво. Покажем, что

$$
|H| \geqslant 2^{k-r-1}
$$

В самом деле, используя правило (3), устанавливаем, что, исходя из произвольного решения системы (11), можно получить совокупность, состоящую из $2^{k-s_{1}+s_{2}+s_{3}}=$ $2^{k-\psi}$ элементов $\left(s_{1}, s_{2}\right.$ и $s_{3}$ введены в доказательстве теоремы 1$)$, принадлежаших конфигурации $H$; кроме того, двум различным решениям системы (11) соответствуют две непересекающиеся равномощные совокупности элементов из $H$. Значит, справедливо неравенство $|H| \geqslant 2^{k-\psi} R$, которое вместе с (13) дает (18).

Покажем, что

$$
|H| \leqslant 2^{k-r-1}
$$

Если $H=\varnothing$, то (19) заведомо выполняется. Пусть $H \neq \varnothing$ и $h \in H$. В силу определения конфигурации $H\left(\omega_{j}\right)$ имеем $\left|h \cap \omega_{j}\right| \equiv 0(\bmod 2), j=\overline{1, \Delta}$. Отсюда на основании (1) и (12) заключаем, что $h \in I_{s}$. Значит, $H \subseteq I_{s}$. Поэтому для произвольного $h \in H$ можно с помощью правила (3) построить решение $\vec{x}(h)$ системы (11) и аналогично $(7)$ убедиться в том, что число элементов из $H$, соответствующих решению $\vec{x}(h)$, не превышает $2^{k-\psi}$. Отсюда получаем неравенство $|H| \leqslant 2^{k-\psi} R$, которое вместе с (13) дает (19).

Соотношения (18) и (19) доказьвают теорему 3. 
4. Пусть $\Omega=\{1, \ldots, k\}$. Рассмотрим матрицу $A$, построенную на $W_{\Delta}$, и матрицу $B$, имеющую $\Delta$ строк и $k$ столбцов, определенную равенством

$$
B=\left\|b_{q}^{(\varkappa)}\right\|_{q=\overline{1, \Delta}}^{\varkappa=\overline{1, k}}
$$

где

$$
b_{q}^{(\varkappa)}=\left\{\begin{array}{ll}
1, & \text { если } \varkappa \in \omega_{q}, \\
0, & \text { если } \varkappa \notin \omega_{q},
\end{array} \quad \varkappa=\overline{1, k}, \quad q=\overline{1, \Delta} .\right.
$$

Лемма 2. Ранги матрии $A$ и В над полем $\mathrm{GF}(2)$ совпадают: $\operatorname{rank}(A)=\operatorname{rank}(B)$.

ДокАЗАТЕЛЬСтво. Пусть элементу $\omega_{j} \in W_{\Delta}$ соответствует по построению строка $\left(a_{j}^{(1)} \ldots a_{j}^{(\varphi)}\right)$ матрицы $A$ и строка $\left(b_{j}^{(1)} \ldots b_{j}^{(k)}\right)$ матрищы $B, j=\overline{1, \Delta}$. Покажем, что линейно независимьм над полем $\mathrm{GF}(2)$ строкам матрицы $A$ соответствуют линейно независимые над $\mathrm{GF}(2)$ строки матрицы $B$, и наоборот. Очевидно, сказанное имеет место, если $\operatorname{rank}(A)=\operatorname{rank}(B)=1$. Пусть $\operatorname{rank}(A)=r, r \geqslant 2$. Не нарушая общности, положим, что первые $r$ строк матрищы $A$ являются линейно независимыми над $\mathrm{GF}(2)$. Тогда строки $\left(b_{j}^{(1)} \ldots b_{j}^{(k)}\right) j=\overline{1, r}$, матрищы $B$ также являются линейно независимыми над $\mathrm{GF}(2)$. Действительно, допустим, что одна из строк, например $\left(b_{r}^{(1)} \ldots b_{r}^{(k)}\right)$, является линейной комбинацией над $\mathrm{GF}(2)$ строк $\left(b_{j}^{(1)} \ldots b_{j}^{(k)}\right) j=\overline{1, r-1}$. В таком случае совокупность $\omega_{r}$ состоит из тех и только тех элементов множества $\Omega$, которыепринадлежат одному или нескольким множествам $\omega_{1}, \ldots, \omega_{r-1}$. Отсюда следует, что $r$-я строка матрицы $A$ является линейной комбинацией над $\mathrm{GF}(2)$ строк с номерами $1, \ldots, r-1$, что противоречит выбору строк $\left(a_{j}^{(1)} \ldots a_{j}^{(\varphi)}\right) j=\overline{1, r}$. Аналогично можно показать, что если $\operatorname{rank}(B)=r$, то $\operatorname{rank}(A)=r$. Лемма 2 доказана.

ТЕОрема 4. Если выполняются условия (1) и

$$
\begin{gathered}
\Delta=2^{r}-1, \quad s=2^{k-r}-1, \quad 1 \leqslant r \leqslant k-2, \\
\left|\omega_{j}\right| \geqslant 3, \quad j=\frac{1}{1, \Delta,}
\end{gathered}
$$

то существует число $\alpha \in\{1, \ldots, \Delta\}$ такое, что для некоторых $m_{i_{\nu}} \in I_{s}, \nu=1,2,3$,

$$
\left|\omega_{\alpha} \cap m_{i_{\nu}}\right|=2, \quad \nu=1,2,3, \quad\left|\omega_{\alpha} \cap(a \cup b)\right|=3,
$$

әде $a \neq b, a, b \in\left\{m_{i_{\nu}}: \nu=1,2,3\right\}$.

ДокАЗАТЕЛьство. В силу $(1),(21)$ и замечания $1 \operatorname{rank}(A)=r$, где $A$ - матрица, построенная на $W_{\Delta}$. Отсюда на основании леммы 2 имеем $\operatorname{rank}(A)=\operatorname{rank}(B)=r$, где $B-$ матрица, определенная равенством (20). Поскольку $B$ состоит из $\Delta=2^{r}-1$ ненулевых строк и $\operatorname{rank}(B)=r$, то первые $r$ строк матрищы $B$ можно выбрать так, чтобы они образовьвали канонический базис $r$-мерного подпространства $k$-мерного пространства над полем $\mathrm{GF}(2)$. Тогда согласно правилу задания канонического базиса (один из вариантов изложения этого правила см., например, в [3, с. 219]) в первых $r$ строках матрицы $B$ существует единичная $(r \times r)$-матрица. Пусть столбцы с номерами $1<a_{1}<\cdots<a_{r} \leqslant k$ образуют указанную $(r \times r)$-матрищу. Применим построенный канонический базис к формированию множеств $m_{i_{1}}, m_{i_{2}}$ и $m_{i_{3}}$, удовлетворяющих (23). В качестве $\alpha$ выберем $\alpha=1$. В силу $(22)$ в первой строке матрицы $B$ на позициях с номерами $j_{1}$ и $j_{2}$ 
расположены единицы, где $j_{1}$ и $j_{2}$ - два целых числа, удовлетворяющих соотношениям $1 \leqslant j_{1}<j_{2}<a_{1}$. Далее воспользуемся методом математической индукции. Пусть $\operatorname{rank}(B)=1$. Тогда в $m_{i_{1}}\left(\right.$ соответственно $\left.m_{i_{2}} ; m_{i_{3}}\right)$ включим лишь следующие элементы: $u_{j_{1}}^{(1)}$ и $u_{j_{2}}^{(1)}$ (соответственно $u_{j_{1}}^{(1)}$ и $u_{a_{1}}^{(1)} ; u_{j_{2}}^{(1)}$ и $\left.u_{a_{1}}^{(1)}\right)$, где $u_{j_{1}}^{(1)}, u_{j_{2}}^{(1)}$ и $u_{a_{1}}^{(1)}$ - элементы множества $\omega_{1}\left(u_{j_{1}}^{(1)}, u_{j_{2}}^{(1)}, u_{a_{1}}^{(1)} \in \omega_{1}\right)$, которые соответствуют по построению единицам первой строки матрищы $B$, расположенньм на позициях с номерами $j_{1}, j_{2}$ и $a_{1}$. Обоснуем индукционньй шаг от $\operatorname{rank}(B)=r-1, r \geqslant 2, \mathrm{k} \operatorname{rank}(B)=r$. Если $\left|m_{i_{\nu}} \cap \omega_{r}\right| \equiv 0(\bmod 2)$, то множество $m_{i_{\nu}}$ оставляем без изменений, иначе дополняем его элементом $u_{a_{r}}^{(r)} \in \omega_{r}$, который соответствует единице, расположенной в $r$-й строке матрицы $B$ на позиции с номером $a_{r}, \nu=1,2,3$. Ясно, что построенное таким образом множество $m_{i_{\nu}}, \nu=1,2,3$, удовлетворяет соотношениям (23) и

$$
\left|m_{i_{\nu}} \cap \omega_{j}\right| \equiv 0(\bmod 2), \quad j \in\{1, \ldots, \Delta\}, \quad \nu=1,2,3
$$

Из (24) следует, что $m_{i_{\nu}} \in I_{s}, \nu=1,2,3$, так как в силу теоремы 1 и условия (21) множество $I_{s}$ содержит все совокупности $m_{1}, \ldots, m_{s}$, для которых вьполняется (1). Теорема 4 доказана.

ЗАмЕчАнИЕ 2. При $r=k-1$ и $s=1$ условие (22) не выполняется. Действительно, в $i$-й строке, $i \in\{1, \ldots, r\}$, канонического базиса на позициях с номерами $j>a_{i}$ стоят нули, а позиции с номерами $j<a_{i}, j \notin\left\{a_{1}, \ldots, a_{i-1}\right\}, i \in\{2, \ldots, r\}$, и $j<a_{1}$ могут быть заполнены элементами поля GF (2) произвольным образом. (Здесь сохранены обозначения, введенные при обосновании теоремы 4.) Поэтому если $r=k-1$ и $s=1$, то существует значение параметра $j \in\{1, \ldots, \Delta\}$ такое, что $\left|\omega_{j}\right|<3$.

\section{СПИСОК ЦИТИРОВАННОЙ ЛИТЕРАТУРЫ}

[1] Тараканов В. Е. Комбинаторные задачи и $(0,1)$-матрицы. М.: Наука, 1985.

[2] Фаддеев Д. К. Лекции по алгебре. М.: Наука, 1984.

[3] Эндрюс Г. Теория разбиений. М.: Наука, 1982. 